\title{
EL PROCESO DE CAMBIO INSTITUCIONAL \\ EN LA ECONOMIA CUBANA
}

Sergio G. Roca

\section{Introducción.}

Con frecuencia, los trabajos académicos sobre Cúba abordan la revolución como un caso sui generis, como un evento único entre los acontecimientos sociales, como un modelo especial. Ello no es así. Cuba es un caso común y debería ser analizado como tal. Entre las opciones posibles como marco de análisis de la economía cubana, se han elegido dos para realizar este trabajo: el modelo económico socialista y la teoría del cambio institucional.

El argumento básico que se examina aborda dos problemas cruciales que afronta la revolución en la hora actual: decadencia y renovación. La decadencia secular de la economía cubana es intrínseca a todas las economías centralmente planificadas. La naturaleza y el proceso de declinación de Cuba son similares a los que tuvieron lugar en anteriores economías centralmente planificadas, pero los aspectos de orden temporal y los detalles son diferentes. La experiencia cubana se ha visto complicada por el colapso de la Unión Soviética y Europa del Este, así como por el alto grado de dependencia económica externa. Este análisis se basa en el modelo de Janos Kornai sobre la declinación económica socialista, que se reseña en The Socialist System. Por otro lado, la evolución potencial hacia la eficiencia económica está relacionada con el ritmo de desarrollo institucional. ¿Es posible caracterizar el proceso de cambio institucional actualmente vigente en Cuba? ¿Cómo ha de ser evaluada esa transformación? Éste estudio se basa en el modelo de Douglas North, que vincula el cambio institucional con el desempeño económico.

El futuro desarrollo de Cuba estará determinado por la interacción entre el proceso de desintegración económica socialista (ya avanzado y cada vez más veloz) y el proceso de cambio institucional 
(de débil tradición y presencia superficial). En este trabajo se examina el camino del deterioro económico socialista (concentrándose en lo ocurrido en el sector privado) y se analizan los datos actuales relativos al desarrollo institucional.

Debe admitirse que es prematuro aludir en este momento a la "mercadización" de la economía de la Cuba socialista. Las autoridades de Cuba, aún fuertemente afianzadas en cuanto a la determinación de la política económica (pero en proceso de perder el control de la actividad económica), hasta ahora han rechazado los esfuerzos de reforma económica, que comprenden la implantación global del sistema de mercado. En efecto, Castro equiparó en el último tiempo la propiedad privada con el robo y juró que Cuba no debía ni pođía repetir los "errores" que llevaron a la disolución del bloque socialista. No obstante, también es cierto que la economía cubana es insostenible, por cuanto depende de un sistema económico fracasado y está privada de asistencia económica extranjera, mientras se esfuerza por ingresar en una economía mundial basada en las reglas del sistema de mercado.

Es concebible que el efecto combinado del fracaso económico sistémico con las fuerzas económicas externas pueda desencadenar un proceso de cambio hacia algún tipo de variante de la economía de mercado. Basándose en las cambiantes condiciones de Cuba desde 1990, es posible visualizar un camino potencial hacia la "mercadización" de la economía.

Este trabajo se compone de tres partes. En la primera, se reseña el marco teórico utilizado en el análisis de la reforma económica durante el socialismo en Cuba, sobre la base de las investigaciones de Janos Kornai; asimismo, se pasa revista someramente a lo ocurrido en el caso cubano. En la segunda, se utiliza el enfoque sobre economía institucional de Douglas North para analizar las perspectivas de los ajustes en Cuba de esta variable crucial. En la tercera, a modo de conclusión, se presentan algunos comentarios relativos a la lucha entre el deterioro y la renovación en la Cuba socialista.

\section{El modelo de Kornai y la reforma cubana.}

La base teórica para examinar el proceso de cambio económico en Cuba está contenida en la obra de Janos Kornai titulada The Socialist 
System. ${ }^{1}$ En este libro, Kornai explica magistralmente cómo las economías de Europa del Este y la Unión Soviética se desmembraron hasta llegar al colapso a causa de sus propias contradicciones internas que, al comienzo, se creyó que podrían resolverse "perfeccionando" el modelo económico socialista. En otras palabras, las fallas sistémicas que en definitiva llevaron a la desintegración del modelo económico socialista fueron objeto, en repetidas oportunidades, de mejoras o "perfeccionamientos", que a su vez contribuyeron a acelerar la desaparición del orden existente.

El modelo de socialismo clásico de Kornai consta de cinco bloques separados, cada uno de cuales está compuesto de elementos esenciales de la estructura económica en orden descendente desde el poder político, pasando por la macrocoordinación, hasta los desequilibrios microeconómicos. El Bloque 1, que contiene "el poder indivisible del Partido Comunista, empapado en su ideología específica", es la clave que explica el resto de los fenómenos sistémicos. Por ello, el Bloque 1 determina la posición dominante de la propiedad estatal (Bloque 2), la preponderancia de la coordinación burocrática (Bloque 3), y así sucesivamente.

Tras examinar la importancia de la coherencia del sistema clásico, Kornai procede a analizar la dinámica de las transformaciones económicas comenzando por la "perfección" del control y continuando con el ascenso del sector privado, la introducción de la autogestión, la implantación del socialismo de mercado, los experimentos realizados con las reformas de precios, y otras iniciativas encaminadas a la renovación sistémica.

En definitiva, el cambio es provocado por las tensiones acumuladas y las contradicciones inevitables del sistema clásico. Kornai identificó "cuatro grupos principales de motivaciones que obligan al cambio de sistema": i) la acumulación de dificultades económicas; ii) la insatisfacción pública; iii) la pérdida de confianza por parte de la élite dirigente; $y$ iv) los acontecimientos ilustrativos registradoš è el ámbito internacional. A fin de cuentas, el fracaso de la reforma -socialista condujo hacia el sistema de mercado.

\footnotetext{
1 Janos Kornai, The Socialist System: The Political Economy of Communism, (Princeton: Princeton University Press, 1992). En la primera mitad del libro, figura un resumen teórico de socialismo clásico (mođelo económico-político de Stalin y Mao) y sus contradicciones internas. En la segunda parte, Kornai aborda los procesos de reforma económica y regresión, especialmente en Hungría y la Unión Soviética. El autor no alude a las transiciones post-socialistas, basánđose en que son procesos que aún están en desarrollo.
} 
El "perfeccionamiento" de la economía socialista de Cuba fue llevado a cabo entre 1976 y 1989, sobre la base de dos modelos económicos absolutamente opuestos. ${ }^{2} \mathrm{El}$ modelo de reforma comenzó en 1976, al aplicarse el Sistema de Dirección y Planificación de la Economía, conocido como SDPE, que procuraba introducir elementos del modelo soviético moderado de reforma económica de los años sesenta.

Una versión limitada, restringida y principalmente fallida del SDPE fue desmantelada en 1985. La fase de reducción de gastos fue introducida por el Proceso de Rectificación con su explícito programa destinado a revitalizar la administración centralizada, las decisiones administrativas y los incentivos morales. En vigor desde 1986 hasta 1989, el Proceso de Rectificación procuraba "rectificar los errores y corregir las tenđencias negativas" de la década anterior, "perfeccionando" así la economía cubana de acuerdo con la perspectiva del modelo de movilización.

El escenario de la liberalización, que es la práctica económica vigente en Cuba, se vio precipitado por las profundas transformaciones de las relaciones económicas socialistas de índole internacional difundidas después de 1989. En otro artículo, he analizado en detalle el proceso de reforma económica de Cuba, en términos del modelo de Kornai, sobre el deterioro de la economía socialista, destacando el ascenso del sector privado. ${ }^{3}$ En esta ocasión bastará un breve resumen para trasmitir el potencial explicativo del modelo de Kornai sobre el caso cubano.

La desaparición de Europa del Este y el colapso de la Unión Soviética fueron los factores claves para forzar cambios limitados en la economía cubana, consistentes en reducir la liberalización. En virtud de éstos, sólo a determinados elementos del mercado les está permitido actuar de manera controlada. Los sectores económicos claves en los que se pone el acento son muy conocidos: el turismo, la

\footnotetext{
${ }^{2}$ Para una reseña global, véase Carmelo Mesa-Lago, The Economy of Socialist Cuba: A Two Decade Appraisal, (Albuquerque: University of New Mexico Press, 1981); Archibald M. Ritter, 1974; y Sergio G. Roca, "Cuban Economic Policy in the 1970s: The Trodden Paths", Studies in Comparative International Development, Spring, 1977. Una perspectiva diferente, figura en Claes Brundenius, Revolutionary Cuba: The Challenge of Economic Growth with Equity, (Boulder, Colorado: Westview Press, 1984); y Andrew Zimbalist y Claes Brundenius, The (cont nota 2) Cuban Economy: Measurement and Analysis of Socialist Performance, (Baltimore: Johns Hopkins, 1989).

${ }^{3}$ Sergio G. Roca, "The End is Near: Why Cuban Socialism Failed", ponencia presentada en las reuniones de la Association for the Study of the Cuban Economy, Miami, Florida, agosto de 1993.
} 
biotecnología y la producción de alimentos. Durante el período 1991-1993, los mecanismos de mercado utilizados en Cuba incluían joint ventures, con inversionistas extranjeros, reforma parcial de los precios de los productos agrícolas, descentralización de las operaciones de comercio exterior, y algunos pasos iniciales hacia la actividad del sector privado en iniciativas relacionadas con los servicios personales.

En el escenario de la liberalización, el régimen político-económico clásico de Cuba ha procurado adaptarse a las nuevas condiciones sin abandonar su rígido modelo económico. La adaptación ha consistido en el uso limitado y reducido de instrumentos similares a los de la economía de mercado, incluida la promoción selectiva de las actividades del sector privado con inversionistas extranjeros. Sin embargo, los cambios constitucionales sobre los derechos de propiedad privada, la descentralización de las operaciones de las empresas de comercio exterior, la expansión autónoma del autoempleo, el crecimiento de la economía paralela o clandestina, la disensión interna entre las élites del poder y la atracción creciente que genera el modelo del mercado en los cuadros directivos de Cuba, indican, de manera evidente, que la prolongada estabilidad del sistema de economía centralmente planificada puede estar en peligro en la Isla.

Durante el otoño de 1989, la reforma de la economía cubana avanzó lentamente por una senda de moderación. Se iniciaron medidas sobre políticas específicas, pero la coordinación macroeconómica siguió siendo débil, por no decir que estuvo totalmente ausente. Entre las nuevas medidas, cabe mencionar: i) la despenalización de la tenencia y gastos en moneda extranjera; ii) la reorganización de la tenencia de la tierra para favorecer la creación de pequeñas parcelas privadas y productores agrícolas independientes; y iii) la extensión del autoempleo legalizado, a fin de incluir decenas de ocupaciones y empleos. La finalidad básica de estas políticas consiste en ampliar el ámbito del sector privado a fin de aumentar la eficiencia de las ganancias a nivel submacroeconómico.

No obstante, los economistas cubanos están muy conscientes de la necesidad de resolver el problema de la falta de un modelo económico coherente y completo. El Centro de Estudios sobre América (CEA), instituto de investigaciones que funciona en La Habana, concluyó en 1993 que "Cuba no ha definido de modo orgánico la manera en que los diferentes sectores económicos deben estar rela- 
cionados", y llamó a "la reorganización de la economía bajo un nuevo sistema administrativo que asegure la integralidad y la coherencia". 4

El Fondo Monetario Internacional fue invitado a La Habana en mayo y noviembre de 1993, "para suministrar a las autoridades cubanas amplia información sobre las experiencias de transición de las ex economías centralmente planificadas en general y, en particular, acerca del papel jugado por el Fondo y el Banco Mundial en ese proceso". 5 El hecho de que la visita se llevó a cabo y el alto nivel de los participantes cubanos (como lo ilustran diversos seminarios con funcionarios de alto rango de la mayoría de los ministerios relacionados con la economía, reuniones con ministros de Comercio Exterior, Precios y Planificación y una reunión con el "zar" económico Carlos Lage), indican lo crucial que es para la Habana la búsqueda de un modelo económico. Los expertos del Fondo concluyeron que "hasta ahora, las autoridades cubanas no poseen un concepto bien definido sobre la magnitud que deberían tener sus esfuerzos de reforma" y expresaron su satisfacción en cuanto a que "los seminarios ciertamente lograron aumentar la conciencia de las autoridades acerca del papel fundamental de las políticas macroeconómicas en el proceso de transacción hacia un visión más orientada al mercado".

Aunque es imposible fijar un plazo, la llegada de la etapa de transición marcaría el comienzo del camino hacia la economía de mercado. En ese momento, la predicción de Kornai sería la siguiente: "el sistema socialista no es capaz de una renovación que pueda liberarlo de sus rasgos disfuncionales, mientras mantenga la exclusiva tutela del Partido Comunista y la dominación del sector estatal". ${ }^{6}$ En otras palabras, hoy es inevitable que "una transformación profunda, duradera y efectiva" del sistema económico y sus mecanismos "requiere un cambio radical... en el sistema político y (en) las relaciones de propiedad". Lo que se deja ver actualmente es un cambio "del" sistema y no en "el" sistema.

¿Qué tipo de transición hacia una variante de la economía de mercado le espera a Cuba? Definitivamente esta monumental pregunta ha de ser contestada de manera conjunta por todos los especialistas dedicados al análisis del tema, en el marco de un conjunto

\footnotetext{
"Julio Carranza Valdés, "Cuba: los retos de la economía", Cuadernos de Nuestra América, septiembre de 1993, pp. 131-159.

${ }^{5}$ Jacques de Groote y FrankMoss, "Winds of Economic Change in Havana", IMF International Report, Washington, D. C., 23 de noviembre de 1993.

${ }^{6} \mathrm{~J}$. Kornai, op. cit., p. 377.
} 
determinado de restricciones nacionales y condiciones internacionales y, además, sujetos a las correspondientes transacciones generadas en la pugna entre los programas político y económico. En esta transformación, el proceso de cambio institucional ciertamente jugaría un papel clave. ¿Qué es el cambio institucional, cómo funciona y cuáles son sus propósitos?

\section{North y el cambio institucional.}

Un conocido modelo de cambio institucional y desarrollo económico es el de Douglas North, especialmente en la forma en que el autor lo reseña en su libro reciente, titulado Institutions, Institutional Change and Economic Performance. ${ }^{7}$ North define las instituciones como "las reglas del juego de una sociedad o, más formalmente, las cotas o límites ideados por los seres humanos que dan forma a la interacción entre ellos. Como consecuencia de lo anterior, éstos estructuran incentivos para el intercambio humano de carácter político, social o económico. El cambio institucional configura la manera en que se desenvuelven las sociedades a través del tiempo, y por ende es la clave para comprender las transformaciones históricas".

Según North, i) el agente del cambio es "el empresario individual que responde a los incentivos encarnados en el marco institucional"; ii) las fuentes del cambio son los cambiantes precios relativos o preferencias; y iii) el proceso de cambio crece de manera arrolladora". Estos elementos aislados se combinan de la siguiente manera para dar inicio al proceso de cambio institucional: "El aumento del cambio proviene de las percepciones de los empresarios que laboran en organizaciones políticas y económicas, en el sentido de que sería preferible que alteraran, en algún grado, el marco institucional existente. Sin embargo, las percepciones dependen fundamentalmente de la información que reciban los empresarios y de la manera en que procesen esa información.... Los cambiantes precios relativos son filtrados a través de los constructos mentales existentes que dan forma a nuestra comprensión de esos cambios de precios. Es obvio que las ideas y la manera en que éstas surten efecto juegan un papel en este fenómeno". De manera más precisa, puede decirse que "si

\footnotetext{
${ }^{7}$ Doiglas North, Institutions, Institutional Change and Economic Performance, (Cambridge, Mass.: Cambridge University Press,1990).
} 
bien los mercados son incompletos, la retroalimentación de la información es, en el mejor de los casos, fragmentaria y los costos de las negociaciones son significativos, luego, los modelos subjetivos de los actores, modificados ambos por esa retroalimentación muy imperfecta y por la ideología, configurarán el camino que ha de seguirse. Entonces, no solamente pueden prevalecer simultáneamente dos caminos divergentes y un desempeño persistentemente mediocre, las percepciones derivadas históricamente de los actores dan forma a las opciones que éstos adoptan".

Si se aplicara el modelo de North a Cuba, esfuerzo algo prematuro y realizado con insuficiente información, puede argumentarse que mientras que los agentes y las fuentes del cambio institucional laboran febrilmente, el proceso de cambio, dado su carácter progresivo, queda considerablemente rezagado. En efecto, Cuba tiene al menos diez años de retardo con respecto a la antigua experiencia mundial sobre reforma económica, incluidos los períodos de progreso y los años de reducción de gastos. Es fácil ampliar el campo de la actividad empresarial en las áreas rurales y en los centros urbanos, pero es mucho más complejo cambiar la estructura de incentivos por el cálculo del riesgo y las ganancias de carácter privado en el ámbito de la sociedad global, especialmente en el contexto de un régimen totalitario.

En Cuba, pese a los alentadores signos de cambio institucional incipiente y potencialmente eficiente, el camino aparece largo y sinuoso. La poderosa presencia de un liderazgo carismático reduce la posibilidad de que surjan actores institucionales y limita sus actividades. En Cuba, las fuentes del cambio institucional (cambios en los precios relativos y/o las preferencias) siguen siendo temas muy mal comprendidos y sospechosos, y han afectado levemente el funcionamiento de la economía interna oficial. La información incompleta y los costos de transacción, fenómenos acentuados por una catastrófica posición económica internacional, complican el proceso de cambio institucional y pueden ampliar la solidez de los "modelos subjetivos".

En verdad, desde 1993, los líderes cubanos han introducido en sus pronunciamientos públicos advertencias cautelosas acerca de las probables reducciones en materia de velocidad y alcance de la reforma económica. En noviembre, Carlos Lage señaló que "hay personas en el extranjero que piensan que Cuba debería elaborar un programa para renovar su sistema económico, pero no se trata de eso; nuestro sistema económico es socialista y nuestra lucha, nuestra determina- 
ción, nuestro esfuerzo consisten en mantener el socialismo". 8 En una reunión de intelectuales cubanos celebrada en ese mismo mes, Castro se quejó, diciendo "hemos tenido que hacer concesiones, es innegable.... Estos cambios eran inevitables y todavía tenemos que hacer otros más que propician el individualismo, el egoísmo y que hacen que el valor del dinero sea más importante en la sociedad, lo cual tiene efectos alienantes; todo esto es un hecho. No es esto lo que queríamos hacer con nuestro país. Queríamos hacer lo que estábamos haciendo en pleno proceso de rectificación cuando ocurrió la catástrofe y sobrevino el colapso". 9

Durante las reuniones de diciembre de la Asamblea Nacional, se introdujo y se debatió con vigor un programa de austeridad económica modesto y ortodoxo (que incluía restricciones monetarias, reducciones del presupuesto y aumentos de precios e impuestos). Castro intervino para exhortar a ser cautelosos y a sostener un mayor debate entre los miembros de la clase trabajadora, advirtiendo que sería difícil mantener un sistema socialista basado solamente en los ingresos provenientes de los impuestos y debilitado por las reformas propuestas. En efecto, el Presidente cubano criticó ásperamente la rápida expansión del floreciente sector privado y probablemente con efecto atemorizador, recordó a los asistentes que las medidas de reforma económica podían ser técnicamente correctas pero políticamente incorrectas. En el primero de varios pasos correctivos, los legisladores redujeron el ámbito de la actividad económica privada dando lugar a una redada de pequeños empresarios en las calles de La Habana.

¿Qué se puede deducir de todo esto? En medio de la declinación económica y al borde del colapso económico, la reforma económica cubana aún es limitada y tentativa, restringida y vacilante, avanza y retrocede. Sin embargo, el tiempo se acaba y debe decidirse qué hacer. Existe consenso en torno a la necesidad de cambio, pero hay un completo desconcierto sobre cómo proceder. Según Pedro Monreal, investigador principal del Centro de Estudios sobre América, "existe acuerdo entre la dirigencia en el sentido de que las cosas tienen que cambiar, pero que no deben cambiar de manera desordenada. Hay una gran dosis de incertidumbre sobre la configuración

\footnotetext{
${ }^{8}$ Granma International, 3 de noviembre de 1993, p. 6.

${ }^{9}$ Discurso de Fidel Castro en el $V$ Congreso de UNEAC, Granma Internacional, 8 de diciembre de 1993, p. 4.
} 
futura de la economía. Se baraja toda clase de propuestas" ${ }^{10}$ Es difícil imaginar que el proceso de cambio institucional pueda llevarse a cabo en Cuba en el futuro cercano.

\section{Resumen y conclusiones.}

En este trabajo, se ha presentado una vía posible para la transformación del sistema económico cubano, teniendo en cuenta los factores que impulsaron su desarrollo desde un escenario de base sociảl socialista hasta un régimen de transición. Las fuerzas que impulsaron esta regeneración sistémica fueron las contradicciones internas de una economía socialista "en fase de perfeccionamiento" y el colapso de la comunidad socialista en el mundo entero. De acuerdo con el modelo de Kornai, la vía cubana al capitalismo -no obstante sus peculiaridades- puede ser visualizada teniendo en cuenta los caminos ya recorridos por Europa de Este y la ex Unión Soviética. En términos del programa de "mercadización", es probable que el camino cubano sea altamente idiosincrásico, reflejando su pasado revolucionario y su situación geopolítica, pero la transición de un sistema orientado al mercado parece estar en marcha. Por otro lado, el aporte del proceso de cambio institucional, en el sentido de North, a la transición del mercado probablemente sea marginal y sectorial. En la Isla, los agentes y las fuerzas del cambio institucional han logrado poco en materia del establecimiento de una base para el consiguiente desarrollo en el momento oportuno. Ahora bien, el momento puede ser adecuado, pero los jugadores son pocos y carecen de experiencia. Es más, el carisma y el control siguen siendo enemigos formidables. Es evidente que el eventual advenimiento de un cambio económico de carácter sistémico en Cuba dará lugar a una proliferación de nuevas investigaciones acerca de sus rasgos -únicos en su género-y de sus especiales características.

${ }^{10}$ The New York Times, 3 de febrero de 1994, p. A4. 